\title{
Intraepidermal Route of Administration
}

National Cancer Institute

\section{Source}

National Cancer Institute. Intraepidermal Route of Administration. NCI Thesaurus. Code C38243.

Administration of a drug within the superficial epithelial layer of the skin. 